\title{
COHOMOLOGICAL DIMENSION OF A GROUP WITH RESPECT TO FINITE MODULES
}

BY

\author{
JUAN JOSE MARTÍNEZ
}

\begin{abstract}
The purpose of this paper is to compare the cohomological dimension of a group, relative to finite modules, with the cohomological dimension, in the usual sense, of its profinite completion. The basic tool used to perform this comparison is certain stable cohomology of the group. The reason is that there exists a spectral sequence which relates the continuous cohomology of the profinite completion, with coefficients in this stable cohomology, to the ordinary cohomology of the group. Moreover, the direct method of connecting the cohomology of the group with the profinite cohomology of its completion arises from the edge effects on the base of this spectral sequence.
\end{abstract}

The problem of comparing dimensions considered here was proposed by Gruenberg [2, \$8.12, p. 177], to whom the author wishes to express his thanks. This paper is divided into two parts. $\$ 1$ deals with the stable cohomology of a group, and contains the results of qualitative nature. In particular, the spectral sequence mentioned above is included in this section, as well as its connection with the comparison method used by Serre in [4, Chapitre I, Exercises du \$2.6, p. I-15]. $\$ 2$ is essentially quantitative. Its heart is the comparison theorem, which is preceded by some preparatory results on dimensions of the three cohomologies involved in the question.

1. Stable cohomology. Throughout this paper, $G$ is an arbitrary group, and $\hat{G}$ denotes the profinite group associated with $G$ [4, Chapitre I, Exemple 3, p. I-1], that is, $\hat{G}=\operatorname{proj} \lim _{U} G / U$, where the limit is taken over all normal subgroups $U$ of finite index in $G$, with respect to the quotient morphisms of the identity map of $G$. Let $h: G \rightarrow \hat{G}$ be the group morphism defined by the projections of $G$ onto its finite quotients $G / U$; explicitly, $h(s)=(s U)_{U}$, for all $s \in G$. Recall that $(\hat{G}, h)$ can be regarded as the separate completion of $G$,

Received by the editors September 19, 1974.

AMS (MOS) subject classifications (1970). Primary 18H10, $20 \mathrm{~J} 05$.

Key words and phrases. Profinite completion of a group, stable cohomology of a group, spectral sequence, cohomological dimension of a group, stable dimension of a group, cohomological dimension of a profinite group. 
with respect to the topology of the subgroups of finite index. The following known relation between subgroups of $G$ of finite index and open subgroups of $G$ will be used repeatedly.

LEMMa 1.1. If $V$ is a subgroup of finite index in $G$, then $\nabla$ is canonically isomorphic to the closure of $h(V)$, which is an open subgroup of $G$. Moreover, any open subgroup $W$ of $\hat{G}$ can be obtained in this way, by taking $V=h^{-1}(W)$.

If $A$ is a $G$-module, $\left(A^{U}\right)_{U}$, where $U$ runs through all normal subgroups of finite index in $G$, is an increasing family of submodules of $A$. Then, $D_{G}(A)=\cup_{U} A^{U}$ is also a $G$-module. Actually, since $G=\operatorname{proj} \lim _{U} G / U$, $D_{G}(A)=\operatorname{ind} \lim _{U} A^{U}$ and $G / U$ operates coherently on $A^{U}, D_{G}(A)$ has a canonical structure of discrete $\hat{G}$-module [3, Chapitre VII, Théorème 3, p. 176]. Hence, $D_{G}$ may be viewed as an additive functor from the category of $G$ modules to the category of discrete $\mathcal{G}$-modules. The right derived cohomological functor of $D_{G}$ is called the stable cohomology of $G$ and is denoted by $H_{s}(G$,$) . (This denomination is justified by 1.3$ and 1.4.) Since $D_{G}$ is left exact, the elementary homological algebra $[2,882.1-2.3, \mathrm{pp} .139-144]$ provides the following

Proposition 1.2. The positive $\partial$-functor $H_{s}(G$,$) is characterized, up to an$ isomorphism, by any one of the following three conditions:

(i) $H_{s}^{*}(G$,$) is universal and H_{s}^{\circ}(G)=,D_{G}$.

(ii) $H_{s}^{\prime}(G$,$) is exact, H_{s}^{\circ}(G)=,D_{G}$, and $H_{s}^{n}(G, Q)=0$, if $n>0$ and $Q$ is an injective G-module.

(iii) $H_{s}^{\prime}(G$,$) is exact, and H_{s}^{\prime}(G, A) \simeq H^{\cdot}\left(D_{G}(C)\right)$, if $A$ is any G-module and $C$ is any injective resolution of $A$.

The stable cohomology of $G$ may be computed by using the ordinary cohomology $H^{\prime}(U$,$) of each normal subgroup U$ of finite index in $G$. To see this, observe that $G / U$ acts on $H^{n}(U, A)$, by conjugation, if $A$ is a $G$-module (cf. [3, Chapitre II, \$1.d, p. 53]; but the notation must be arranged via the inversion map $s \mapsto s^{-1}$ of $G$ ). Under these actions, the quotient morphism $G / V \rightarrow G / U$ and the restriction morphism $H^{n}(U, A) \rightarrow H^{n}(V, A)$ are compatible, whenever $U \supseteq V$ [3, Chapitre II, Proposition 7, p. 54]. Therefore, ind $\lim _{U} H^{n}(U, A)$, where the limit is taken with respect to the restrictions, becomes a discrete $\hat{G}$-module, again by [3, Chapitre VII, Théorème 3, p. 176]. Then, regarding $H^{n}(U$,$) as a functor from G$-modules to $G / U$-modules, ind $\lim _{U} H^{n}(U$,$) becomes a functor from G$-modules to discrete $G$-modules. Thus, the corresponding cohomological functor ind $\lim _{U} H^{*}(U$,$) is defined$ for $G$-modules, with values in the category of discrete $G$-modules. Now, let $Q$ be an injective $G$-module. Since $Q$ is $S$-injective, for all subgroups $S$ of $G$, because the group ring $Z[G]$ is free (and hence, projective), as a $S$-module, it 
follows that ind $\lim _{U} H^{n}(U, Q)=0$, if $n>0$. Moreover, ind $\lim _{U} H^{\circ}(U$, ) $=D_{G}$, for $H^{\circ}(U, A)=A^{U}$, as $G / U$-modules. Therefore, 1.2, (ii) applies to give the

COROLLARY 1.3. There is an isomorphism of $\partial$-functors

$$
H_{s}(G,) \simeq \text { ind } \lim _{U} H^{\cdot}(U,) .
$$

Alternatively, regard $H^{n}(U$,$) as a functor on G$-modules to discrete $G$ modules, via the canonical morphism $\hat{G} \rightarrow G / U$. Then,

$$
D_{G}=\text { ind } \lim _{U} H^{\circ}(U,)
$$

and $H^{n}(U$,$) is the right n$th derived of $H^{\circ}(U$,$) . Hence, the corollary also$ follows from [1, Proposition 3.10.1, p. 181].

The stable cohomology does not change, when $G$ is replaced by any subgroup of finite index. In a precise manner,

Proposition 1.4. Let $V$ be a subgroup of finite index in $G$, and let $R_{V}$ be the restriction functor on $G$-modules to $V$-modules. Then, if $A$ is a $G$-module, the action of $\hat{\nabla}$ on $H_{s}^{n}(V, A)$ can be extended to $\hat{G}$, so that $H_{s}\left(V, R_{V}()\right)$ becomes a cohomological functor isomorphic to $H_{s}(G$,$) .$

Proof. The normal subgroups $U$ of $G$, of finite index, contained in $V$ form a final subset of the set of normal subgroups of $V$, of finite index, and so,

$$
H_{s}^{n}(V, A) \simeq \underset{U \subseteq V}{\operatorname{ind} \lim } H^{n}(U, A),
$$

not only as abelian groups, but as discrete $\nabla$-modules, since $\nabla$ $\simeq$ proj $\lim V / U(U \subseteq V)$. But, a fortiori, this indexing set is final in the set of all normal subgroups of finite index in $G$. Thus,

$$
\dot{G} \simeq \operatorname{proj} \lim _{U \subseteq V} G / U \text {. }
$$

By means of these two isomorphisms, $H_{s}^{n}(V, A)$ becomes a discrete $\hat{G}$-module, as asserted. Furthermore, there is an isomorphism of discrete $\hat{G}$-modules

$$
H_{s}^{n}(G, A) \simeq \underset{U \subseteq V}{\operatorname{ind} \lim } H^{n}(U, A),
$$

again by finality. Hence, $H_{s}^{n}(V, A) \simeq H_{s}^{n}(G, A)$, as discrete $\hat{G}$-modules, and consequently

$$
H_{s}^{*}\left(V, R_{V}()\right) \simeq H_{s}^{*}(G,) .
$$

Assume that $A^{V}=A$. (For example, if $A$ is finite, this condition is fulfilled by taking $V$ as the stabilizer of $A$ in $G$.) Then, if $T(A)$ denotes $A$ viewed as a 
trivial $G$-module, $H^{n}(U, A) \simeq H^{n}(U, T(A))$, as $G / U$-modules, for $U$ contained in $V$, because $A$ and $T(A)$ are isomorphic $U$-modules. Therefore, passing to the limit over $U$,

$$
H_{s}^{n}(G, A) \simeq H_{s}^{n}(G, T(A)),
$$

and the following is proved, for later use,

Proposinion 1.5. The cohomological functors $H_{s}(G$,$) and H_{s}(G, T())$ are isomorphic on the category of G-modules $A$ satisfying $A^{V}=A$, for some subgroup $V$ of finite index in $G$. In particular, they are isomorphic on the category of finite G-modules.

The next proposition says that the functor $D_{G}$ preserves "isogenies".

Proposinion 1.6. If $f: A \rightarrow B$ is an epimorphism of G-modules, with finite kernel, then $D_{G}(f): D_{G}(A) \rightarrow D_{G}(B)$ is also an epimorphism. Equivalently, $H_{s}^{1}(G, C)=0$, for all finite G-modules $C$.

Proof. Take $y \in B^{U}, U$ being a normal subgroup of finite index in $G$, and write $y=f(x)$, with $x \in A$. Since $s x-x \in \operatorname{Ker} f$, for $s \in U$, the identity $s x=x+(s x-x)$ shows that $U x$ is finite. Hence it follows that $G x$ is finite, since $U$ has finite index in $G$. Therefore, observing that $D_{G}(A)$ consists of all elements in $A$ whose orbits are finite, $x \in D_{G}(A)$. Thus, $D_{G}(f)$ is an epimorphism.

Finally, the equivalence follows from the fact that $H_{s}^{1}(G$,$) is the right first$ derived functor of $D_{G}$.

The main result of this section establishes that the continuous cohomology $H_{c}^{*}(\hat{G}$,$) of \hat{G}$, combined with the stable cohomology of $G$, approximate the usual cohomology of $G$.

THEOREM 1.7. There exists a cohomological spectral functor on the category of G-modules yielding the convergence

$$
H_{c}^{p}\left(\hat{G}, H_{s}^{q}(G, A)\right) \Rightarrow H^{n}(G, A) .
$$

Proof. Note that

$$
H_{c}^{\circ}\left(\hat{G}, H_{s}^{\circ}(G,)\right)=H^{\circ}(G,),
$$

for $D_{G}(A)^{G}=\cup_{U}\left(A^{U}\right)^{G / U}=A^{G}$. Hence, applying the spectral theorem of composite functors [1, Theorème 2.4.1, p. 148], it suffices to show that $D_{G}$ preserves injectives. But this follows from the fact that $D_{G}$ has a left adjoint, which is exact, namely, the functor $F_{G}$ obtained by pulling back the operators along the morphism $h: G \rightarrow \hat{G}$. In fact, if $M$ is a discrete $\hat{G}$-module, the 
inclusion of $D_{G}(A)$ in $A$ induces a group injection

$$
\operatorname{Hom}_{G}\left(M, D_{G}(A)\right) \rightarrow \operatorname{Hom}_{G}(M, A),
$$

which is natural in $(M, A)$. But, further, it is surjective, since the image of a $G$ morphism of $M$ into $A$ is contained in $D_{G}(A)$. (For, given such a morphism $f$, if $x \in M^{W}$, where $W$ is a normal, open subgroup of $G$, then, taking $V=h^{-1}(W), V$ is a normal subgroup of finite index in $G$ such that $f(x)$ $\in A^{V}$.) Therefore,

$$
\operatorname{Hom}_{G}\left(, D_{G}()\right) \simeq \operatorname{Hom}_{G}\left(F_{G}(),\right),
$$

as asserted. Finally, it is clear that $F_{G}$ is an exact functor.

Alternative Proof. [4, Chapitre I, Proposition 8, p. I-9], together with 1.3, show that

$$
H_{c}^{p}\left(\hat{G}, H_{s}^{q}(G, A)\right) \simeq \text { ind } \lim _{U} H^{p}\left(G / U, H^{q}(U, A)\right)
$$

But,

$$
H^{p}\left(G / U, H^{q}(U, A)\right) \Rightarrow H^{n}(G, A),
$$

by using the Hochschild-Serre spectral sequence $(E(U, A), H(A))[3$, Chapitre VI, \&2, p. 158]. Moreover, the family $((E(U, A), H(A)))_{U}$ has a structure of direct system (of spectral sequences) such that, for $U \supseteq V$, the morphism $E_{2}^{p, q}(U, A) \rightarrow E_{2}^{p, q}(V, A)$ coincides with the morphism $H^{p}\left(G / U, H^{q}(U, A)\right)$ $\rightarrow H^{p}\left(G / V, H^{q}(V, A)\right)$ belonging to the direct system above, and the morphism $H^{n}(A) \rightarrow H^{n}(A)$ is the identity. The theorem follows, now, by taking the limit.

COROllary 1.8. If $A$ is a finite G-module, then

$$
\begin{aligned}
& H_{c}^{1}\left(\hat{G}, D_{G}(A)\right) \stackrel{\sim}{\longrightarrow} H^{1}(G, A), \\
& H_{c}^{2}\left(\hat{G}, D_{G}(A)\right) \stackrel{\subset}{\longrightarrow} H^{2}(G, A) .
\end{aligned}
$$

Proof. The spectral sequence associated with $A$ satisfies $E_{2}^{0,1}=0$, by 1.6. Thus, the result follows from the corresponding exact sequence for the low degrees.

REMARK 1.9. In [4, Chapitre I, Exercises du \$2.6, p. I-15] Serre establishes relations between discrete cohomology and profinite cohomology. The spectral functor provided by 1.7 can be applied to Serre's comparison method. To perform this application, it is necessary to determine the edge effects on the base. 
Let $A$ be a $G$-module. If $U$ and $V$ are normal subgroups of finite index in $G$ and $U \supseteq V$, the diagram

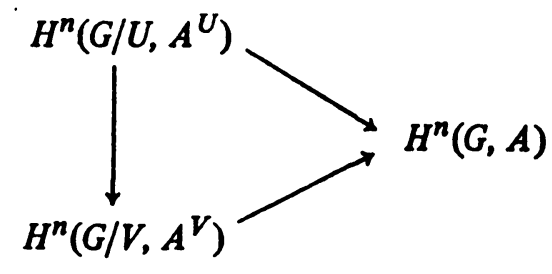

where the morphisms are the inflations, commutes [3, Chapitre II, Proposition 5, p. 53]. Therefore, using [4, Chapitre I, Proposition 8, p. I-9], the family of inflations $\left(H^{n}\left(G / U, A^{U}\right) \rightarrow H^{n}(G, A)\right)_{U}$ yields a morphism

$$
H_{c}^{n}\left(\hat{G}, D_{G}(A)\right) \rightarrow H^{n}(G, A)
$$

Since the inflation $H^{n}\left(G / U, A^{U}\right) \rightarrow H^{n}(G, A)$ is the edge morphism on the base of the Hochschild-Serre spectral sequence of $(U, A)[3$, Chapitre VI, Proposition 2, p. 160], then $H_{c}^{n}\left(\hat{G}, D_{G}(A)\right) \rightarrow H^{n}(G, A)$ is the edge morphism on the base of the spectral sequence corresponding to $A$, by the alternative proof of 1.7. (Analogously, the morphism $H^{n}(G, A) \rightarrow H_{s}^{n}(G, A)^{G}$ induced by the canonical morphism $H^{n}(G, A) \rightarrow H_{s}^{n}(G, A)$, for the image of this lies in $H_{s}^{n}(G, A)^{G}$, is the edge morphism on the fiber of the spectral sequence of $A$.) Explicitly, $H_{c}^{n}\left(\hat{G}, D_{G}(A)\right) \rightarrow H^{n}(G, A)$ may be described as follows: the image of the cohomology class represented by a cocycle $f$ is the cohomology class of the cocycle $i f h^{n}, i$ being the inclusion of $D_{G}(A)$ in $A$. This fact is easy to verify by means of the explicit expression of the inflation [3, Chapitre II, \$1.c, p. 50].

Furthermore, observe that the composition $D_{G} F_{G}$ is the identity functor on the category of discrete $\hat{G}$-modules. In fact, if $M$ is such a module, $D_{G}(M)$ $=U_{U} M^{h(U)}$; but $M^{h(U)}=M^{\overline{h(U)}}$, since $\hat{G}$ operates continuously on $M$. Hence, applying 1.1, $D_{G}(M)=\cup_{V} M^{V}$, where $V$ runs through all normal, open subgroups of $G$, and $M$ being discrete, $\cup_{V} M^{V}=M$.

Now, if $M$ is a discrete $\hat{G}$-module, making $A=F_{G}(M)$ in $H_{c}^{n}\left(\hat{G}, D_{G}(A)\right)$ $\rightarrow H^{n}(G, A)$ provides a morphism

$$
H_{c}^{n}(\hat{G}, M) \rightarrow H^{n}(G, M),
$$

which sends the cohomology class represented by a cocycle $f$ to the cohomology class of the cocycle $f h^{n}$. Hence, this morphism coincides with that deduced from the morphism of cohomological functors

$$
H_{c}^{\prime}(\hat{G},) \rightarrow H^{\cdot}\left(G, F_{G}()\right)
$$

extending the identity $H_{c}^{\circ}(\hat{G},) \rightarrow H^{\circ}\left(G, F_{G}()\right)$ (recall that $H_{c}(\hat{G}$, ) is a 
universal $\partial$-functor). But this morphism of cohomological functors is that used by Serre in [4, Chapitre I, Exercises du §2.6, p. I-15]. Therefore,

If $H_{s}^{q}(G, M)=0$, for $0<q \leqslant n$ and for all finite, discrete $\hat{G}$-modules $M$, then $G$ satisfies $A_{n}$. In particular, $G$ satisfies $A_{1}$.

Proof. The spectral sequence of such a module $M$ verifies $E_{2}^{p, q}=0$, for $0<q<n$. Consequently, the edge morphism $E_{2}^{p, 0} \rightarrow H^{p}$ is bijective; for $p<n$, and injective, for $p=n+1$. Moreover, 1.4 shows that the hypothesis of the proposition is fulfilled in the case $n=1$.

2. Cohomological dimensions. If $A$ is a finite $G$-module, then $H^{n}(G, A)$ is a torsion abelian group. Indeed, $H^{n}(G, A)$ is annihilated by some integer $\neq 0$, because $A$ is. Consequently, the adequate version, for the discrete case, of the cohomological dimension theory of profinite groups [4, Chapitre I, 83, p. I-17] is obtained adopting the following definitions.

Let $p$ be a prime number. The cohomological dimension of $G$, with respect to the category of finite, $p$-primary $G$-modules (in the sense of $[2,88.12, p$. 175]), is called the (cohomological) p-dimension of $G$, and is denoted by $d_{p}(G)$. The (cohomological) dimension $d(G)$ of $G$ is defined by considering the category of all finite $G$-modules.

Note that $d(G)=\sup _{p} d_{p}(G)$, where $p$ runs over all prime numbers. Of course, this equality follows from the decomposition of a torsion abelian group into its p-primary components.

The arguments used in [4, Chapitre I, Proposition 14, p. I-20] can easily be adapted to prove the following auxiliary result.

Proposition 2.1. Let $p$ be a prime, and let $V$ be a subgroup of finite index in G. Then:

(i) $d_{p}(V) \leqslant d_{p}(G)$.

(ii) If $d_{p}(G)$ is finite, $d_{p}(V)=d_{p}(G)$.

The stable p-dimension $s d_{p}(G)$ of $G$, where $p$ is a prime, and the stable dimension $\operatorname{sd}(G)$ of $G$ are defined by using $H_{s}^{*}(G$,$) instead of H^{*}(G$,$) .$

It is interesting to observe that $\mathbf{Z}_{p}=\mathbf{Z} / p \mathbf{Z}$, with the trivial $G$-action, is a "test module" for estimating $s d_{p}(G)$, in the following sense: $s d_{p}(G)<n$, where $n$ is a nonnegative integer, if (and only if) $H_{s}^{r}\left(G, \mathbf{Z}_{p}\right)=0$, for all integers $r>n$. In fact, if $A$ is a finite $G$-module annihilated by $p$, then the trivial $G$ module $T(A)$ is isomorphic to a finite direct sum $\mathbf{Z}_{p}^{(I)}$. Hence, applying 1.5 , it follows that $H_{s}^{r}(G, A)=0$, for $r>n$, and arguing by induction on the order, the same holds for $A$ p-primary.

Of course, $s d(G)=\sup _{p} s d_{p}(G)$, while the next result corresponds to 2.1 .

Proposition 2.2. Let $p$ be a prime, and let $V$ be a subgroup of finite index in G. Then, $s d_{p}(V)=s d_{p}(G)$. 
Proof. For any $G$-module $A, H_{s}^{r}(G, A) \simeq H_{s}^{r}(V, A)$, by 1.4. Hence, taking $A$ finite and $p$-primary, it follows that $s d_{p}(G) \leqslant s d_{p}(V)$.

On the other hand, let $B$ be a finite $V$-module. If $B^{\prime}$ is $B$ considered as a trivial $G$-module. then $H_{s}^{r}\left(G, B^{\prime}\right) \simeq H_{s}^{r}\left(V, B^{\prime}\right)$, again by 1.4. But, applying 1.5, $H_{s}^{r}\left(V, B^{\prime}\right) \simeq H_{s}^{r}(V, B)$, since $B$ is finite. Therefore, $H_{s}^{r}(V, B) \simeq H_{s}^{r}\left(G, B^{\prime}\right)$, and so, considermy $B$ p-primary, $s d_{p}(V) \leqslant s d_{p}(G)$.

Finally, a $r_{1}$ will will be needed concerning discrete modules over profinite groups.

Let $A$ be a $G$-module. $A_{G}$ denotes $A / I_{G} \cdot A, I_{G}$ being the augmentation ideal of $G$. Recall that $A_{G}$ is the largest quotient module on which $G$ acts trivially.

If $p$ is a prime and $K$ is a profinite group, $c d_{p}(K)$ is the cohomological $p$ dimension of $K$, in the usual sense [4, Chapitre I, \$3.1, p. I-17].

Proposition 2.3. Let $K$ be a profinite group, and let $M$ be a discrete $K$-module. Then:

(i) The family $\left(M_{U}\right)_{U}$, where $U$ runs through all normal, open subgroups of $K$, is an inverse system, with respect to the quotient morphisms of the identity map of $M$. The morphism $M \rightarrow \operatorname{proj} \lim _{U} M_{U}$ induced by the family of projections $\left(M \rightarrow M_{U}\right)_{U}$ is an injection. Hence, if $M \neq 0$, each normal, open subgroup $U$ of $K$ contains one, say $V$, such that $M_{V} \neq 0$.

(ii) Assume that, for a prime $p, n=c d_{p}(K)$ is finite, $H_{c}^{n}\left(K, \mathbf{Z}_{p}\right) \neq 0$ and $M$ is annihilated by a pr,wer of $p$. Then, $M_{K} \neq 0$ implies $H_{c}^{n}(K, M) \neq 0$.

Proof. (i) The kernel of the morphism $M \rightarrow \operatorname{proj} \lim _{U} M_{U}$ is $\cap_{U} I_{U} \cdot M$. Since $M$ is the increasing union of $M^{V}$, where $V$ runs also through the set of normal, open subgroups of $K$, then $I_{U} \cdot M=\cup_{V} I_{U} \cdot M^{V}$. Thus,

$$
\bigcap_{U} I_{U} \cdot M=\bigcap_{U}\left(\bigcup_{V} I_{U} \cdot M^{V}\right)=\bigcup_{V}\left(\bigcap_{U} I_{U} \cdot \dot{M}^{V}\right)=0
$$

for $I_{V} \cdot M^{V}=0$.

(ii) Since $M$ is annihilated by a power of $p, M_{K}$ inherits this property, via the projection of $M$ onto $M_{K}$. Then, provided that $M_{K} \neq 0$, there is an epimorphism $M_{K} \rightarrow \mathrm{Z}_{p}$, by the elementary theory of abelian groups. The projection of $M$ onto $M_{K}$, followed by the preceding morphism, yield an epimorphism $M \rightarrow \mathbf{Z}_{p}$. But the induced morphism $H_{c}^{n}(K, M) \rightarrow H_{c}^{n}\left(K, \mathbf{Z}_{p}\right)$ is also surjective, because $H_{c}^{n}(K$,$) is right exact on the category of p$-primary, discrete $K$ modules [4, Chapitre I, Proposition 11, p. I-17]. Therefore, $H_{c}^{n}(K, M) \neq 0$, since $H_{c}^{n}\left(K, \mathbf{Z}_{p}\right) \neq 0$.

The second part of the preceding proposition generalizes [4, Chapitre I, Corollaire à la Proposition 21, p. I-32]. Let $K$ be a pro-p-group, and let $n=c d_{p}(K)$ be finite. (Of course, $H_{c}^{n}\left(K, \mathbf{Z}_{p}\right) \neq 0$, by [4, Chapitre I, Proposition 21, p. I-32].) Further, let $M$ be a discrete $K$-module, which is finite and $p$ - 
primary. If $U$ is a normal, open subgroup of $K$ acting trivially on $M$, then $M_{K}=M_{F}$, where $F=K / U$ is a finite $p$-group. But $M \neq 0$ implies $M_{F} \neq 0$, applying [3, Chapitre III, Proposition 1, p. 81] to the dual module $N$ $=\operatorname{Hom}\left(M, \mathbf{Z}_{p}\right)$ of $M$, where $M$ is regarded as an $F$-module, because $N^{F}$ $=\operatorname{Hom}_{F}\left(M, \mathbf{Z}_{p}\right)=\operatorname{Hom}\left(M_{F}, \mathbf{Z}_{p}\right)$.

CoRollary 2.4. Suppose that, for a prime $p, n=c d_{p}(K)$ is finite and $M$ is annihilated by a power of $p$. Then, if $M \neq 0$, there exists a normal, open subgroup $U$ of $K$ such that $H_{c}^{n}(U, M) \neq 0$.

Proof. Let $N$ be a discrete $K$-module, simple and $p$-primary, such that $H_{c}^{n}(K, N) \neq 0$ [4, Chapitre I, Proposition 11, p. I-17]. Since $N$ is discrete and simple, there is a normal, open subgroup $U$ of $K$ acting trivially on $N$. Further, taking $U$ sufficiently small, it can be assumed that $M_{U} \neq 0$, by 2.3 , (i). Since $N$ is annihilated by $p$, because it is simple and $p$-primary, and $U$ operates trivially on $N$, then $N \simeq \mathbf{Z}_{p}^{(I)}$, for some (finite) set $I$, as $U$-modules. Hence, $H_{c}^{n}(U, N) \simeq H_{c}^{n}\left(U, \mathbf{Z}_{p}\right)^{(I)}$. But $H_{c}^{n}(K, N) \neq 0$ implies $H_{c}^{n}(U, N) \neq 0$, because there is an epimorphism $H_{c}^{n}(U, N) \rightarrow H_{c}^{n}(K, N)[4$, Chapitre I, Lemme 4, p. I-20]. Therefore, $H_{c}^{n}\left(U, \mathbf{Z}_{p}\right) \neq 0$, and applying to $U 2.3$, (ii), $H_{c}^{n}(U, M)$ $\neq 0$, since $M_{U} \neq 0$.

Now, the main result of this paper will be established.

THEOREM 2.5. The following statements are true:

(i) $s d_{p}(G) \leqslant d_{p}(G) \leqslant s d_{p}(G)+c d_{p}(\hat{G})$, for all prime numbers $p$, and so, $s d(G) \leqslant d(G) \leqslant s d(G)+c d(\hat{G})$.

(ii) If $p$ is a prime number such that $s d_{p}(G)$ and $c d_{p}(\hat{G})$ are finite, then $d_{p}(G)=s d_{p}(G)+c d_{p}(\hat{G})$. Hence, $d(G)=s d(G)+c d(\hat{G})$, provided that $\operatorname{sd}(G)$ and $c d(\hat{G})$ are both finite.

Proof. (i) The left-hand inequality, $s d_{p}(G) \leqslant d_{p}(G)$, follows from 1.3 and 2.1, excluding, previously, the trivial case $d_{p}(G)=\infty$.

To prove the right-hand inequality, $d_{p}(G) \leqslant s d_{p}(G)+c d_{p}(\hat{G})$, assume that $m=s d_{p}(G)$ and $n=c d_{p}(\hat{G})$ are finite (otherwise, the inequality is trivial.) If $A$ is a finite, $p$-primary $G$-module, the spectral sequence $(E, H)$ associated with $A$ in 1.7 satisfies, for $i+j>n+m$,

$$
E_{2}^{i, j} \simeq H_{c}^{i}\left(\hat{G}, H_{s}^{j}(G, A)\right)=0
$$

because $i>n$ or $j>m$. Therefore, if $k>n+m$,

$$
H^{k} \simeq H^{k}(G, A)=0
$$


by the maximum principle for spectral sequences.

(ii) This principle also yields an isomorphism $E_{2}^{n, m} \simeq H^{n+m}$, that is,

$$
H_{c}^{n}\left(\hat{G}, H_{s}^{m}(G, A)\right) \simeq H^{n+m}(G, A) .
$$

Thus, $d_{p}(G)=n+m$ if (and only if) there exists a finite, $p$-primary $G$-module $A$ such that $H_{c}^{n}\left(\hat{G}, H_{s}^{m}(G, A)\right) \neq 0$. On the other hand, in view of 2.1, the equality to be proved follows from showing that $d_{p}(V)=n+m$, for some subgroup $V$ of finite index in $G$. But $n=c d_{p}(\hat{V})$, by [4, Chapitre I, Proposition 14, p. I-20], since $\nabla$ is isomorphic to an open subgroup of $G$ (cf. $1.1)$, and $m=s d_{p}(V)$, by 2.2. Therefore, in the proof, $G$ can be replaced by any subgroup of finite index, without loss of generality.

Now, let $A$ be a finite, $p$-primary $G$-module such that $H_{s}^{m}(G, A) \neq 0$. According to $2.4, H_{c}^{n}\left(W, H_{s}^{m}(G, A)\right) \neq 0$, for some normal, open subgroup $W$ of $\hat{G}$. Further, putting $V=h^{-1}(W), 1.1$ says that $V$ is a normal subgroup of finite index in $G$ such that $\hat{V} \simeq W$. Hence,

$$
H_{c}^{n}\left(W, H_{s}^{m}(G, A)\right) \simeq H_{c}^{n}\left(\hat{\nabla}, H_{s}^{m}(V, A)\right),
$$

by 1.4 . In conclusion, changing $V$ by $G$,

$$
H_{c}^{n}\left(\hat{G}, H_{s}^{m}(G, A)\right) \neq 0
$$

as was to be proved.

COROllary 2.6. Assume that, for a prime $p, c d_{p}(\hat{G})$ is finite. Then:

(i) $c d_{p}(\hat{G}) \leqslant d_{p}(G)$.

(ii) $c d_{p}(\hat{G}) \neq d_{p}(G)$ implies $c d_{p}(\hat{G})+1<d_{p}(G)$.

Moreover, the proposition obtained by omitting $p$ is also true.

Proof. Apply 2.5, and notice that $s d_{p}(G) \neq 1$ (always), by 1.6 .

The $G$-modules $A$ satisfying $F_{G} D_{G}(A)=A$ are called discrete. Since $D_{G} F_{G}$ is the identity functor on the category of discrete $\hat{G}$-modules, this category is isomorphic to the category of discrete $G$-modules, in the preceding sense.

Let $g$ be the cohomological dimension of $G$, with respect to the category of discrete $G$-modules, which are torsion abelian groups $[2,88.12$, p. 175]. Since this category contains the finite $G$-modules, $d(G) \leqslant g$. Thus, the corollary shows that $c d(\hat{G}) \leqslant g$, when $c d(\hat{G})$ is finite. This inequality is an answer to the question suggested by Gruenberg in $[2, \$ 8.12$, p. 177].

\section{REFERENCES}

1. A. Grothendieck, Sur quelques points d'algèbre homologique, Tôhoku Math. J. 9 (1957), 119-221. MR 21 \#1328. 
2. K. W. Gruenberg, Cohomological topics in group theory, Lecture Notes in Math., vol. 143, Springer-Verlag, Berlin and New York, 1970. MR 43 \#4923.

3. S. Lang, Rapport sur la cohomologie des groupes, Math. Lecture Note Ser., No. 5808, Benjamin, New York, 1967. MR 35 \#2948.

4. J.-P. Serre, Cohomologie galoisienne, Lecture Notes in Math., vol. 5, Springer-Verlag, Berlin and New York, 1965. MR 34 \# 1328.

Departamento de Matematicas, Facultad de Cienclas Exactas y Naturales, Universidad de Buenos Aires, Buenos Aires, Argentina 